\section{THU0286 RECURRENCE OF ARTERIAL AND VENOUS THROMBOSES IN PRIMARY ANTIPHOSPHOLIPID SYNDROME}

G. Balbi, F. Signorelli, R.A. Levy. Rheumatology, Universidade do Estado do Rio de Janeiro, Rio de Janeiro, Brazil

Background: Antiphospholipid syndrome (APS) is the thrombophilia associated with the highest risk of recurrence of both arterial and venous thromboses. $(1,2)$ In addition to the standard of care (lifelong oral anticoagulation), identification of other risk factors is important to provide better care and to reduce the incidence of rethrombosis.

Objectives: To investigate the risk factors associated with recurrence of arterial and venous thromboses in primary APS patients.

Methods: A cross-sectional study was performed in a group of 80 outpatients who fulfilled APS classification criteria (Sydney). The patients were regularly seen in our department, and clinical and serological features were collected during visits and by chart review. They were classified as recurrent or not, and these groups were compared. Recurrence was defined as the presence of 2 or more thrombotic events during lifetime. Patients with 1 thrombotic event were classified as "no recurrent". No history of thrombosis was the only exclusion criteria.

Results: Of 80 pAPS patients, thirty-five had arterial thrombosis and 54 had venous thrombosis. Of them, thirty-six had recurrent thromboses (11 arterial and 25 venous). Demographic and clinical characteristics of the arterial group are shown in Table 1. In a bivariate analysis, recurrent arterial thromboses were associated with hypertension $(\mathrm{p}=0.016)$ and positivity to lupus anticoagulant (LA; $p=0.033$ ), and recurrent venous thrombosis correlated to obesity $\left(B M l \geq 30 \mathrm{~kg} / \mathrm{m}^{2}\right.$; $p=0.003$ ). In a multivariate regression analysis, the model of recurrent arterial thromboses was adjusted to age, sex, and variables with $\mathrm{p}<0.10$ in the bivariate analysis (age, hypertension, dyslipidemia, and positivity to lupus anticoagulant). Hypertension (OR 9.02; Cl95\% 1.05-77.5; $p=0.045$ ) and age (OR 1.16; Cl95\% $1.01-1.33 ; p=0.035)$ increased the risk of relapse of arterial thrombosis.

Table 1. Demographic and clinical characteristics of patients with recurrent and non-recurrent arterial thromboses $(\mathrm{N}=35)$

\begin{tabular}{lcc}
\hline Variable & Arterial recurrence $(\mathrm{N}=11)$ & No arterial recurrence $(\mathrm{N}=24)$ \\
\hline Age & $53.5 \pm 7.7$ & $41.9 \pm 11.7$ \\
Female gender & $10(90.9)$ & $18(75)$ \\
Caucasian & $8(72.7)$ & $14(58.3)$ \\
Time first manifestation $(\mathrm{mo})$ & $185(120-300)$ & $150(59,3-204)$ \\
Risk factors & & \\
$\quad$ Hypertension & $8(72.7)$ & $7(29.2)$ \\
$\quad$ Diabetes & $1(9.1)$ & $1(4.2)$ \\
Dyslipidemia & $8(72.7)$ & $9(37.5)$ \\
Obesity (BMl $\left.\geq 30 \mathrm{~kg} / \mathrm{m}^{2}\right)$ & $4(36.4)$ & $7(29.2)$ \\
Premature CVD & $3(27.3)$ & $2(8.3)$ \\
$\quad$ Smoking & $5(45.5)$ & $9(37.5)$ \\
Non criteria & & \\
$\quad$ Livedo & $4(36.4)$ & $9(37.5)$ \\
Thrombocytopenia & $1(9.1)$ & $4(16.7)$ \\
Valvulopathy & $1(11.1)^{\star}$ & $2(13.3)^{\star \star}$ \\
Raynaud phenomenon & $4(36.4)$ & $6(25.0)$ \\
Migraine & $6(54.5)$ & $12(50.0)$ \\
\hline
\end{tabular}

${ }^{*} \mathrm{~N}=9 ;{ }^{* *} \mathrm{~N}=15 . \mathrm{Mo}=$ months. CVD = cardiovascular disease. Values showed as $\mathrm{N}(\%)$ for categorical variables, Mean \pm SD for normal distribution and Median (interquartil range) for asymmetric distribution.

Conclusions: This data suggests that hypertension, a traditional cardiovascular risk factor, is associated with increased risk of recurrent thrombosis and, therefore, should be controlled in pAPS patients. Besides, each additional year of age is associated with the risk of $16 \%$ of a new arterial thrombotic event.

References:

[1] R. Cervera, M.A. Khamashta, Y. Shoenfeld, et al., Morbidity and mortality in the antiphospholipid syndrome during a 5-year period: a multicentre prospective study of 1000 patients, Ann. Rheum. Dis. 68 (2009) 1428-1432.

[2] M. Turiel, P. Sarzi-Puttini, R. Peretti, et al., Thrombotic risk factors in primary antiphospholipid syndrome: a 5-year prospective study, Stroke 36 (2005) 1490-1494.

Disclosure of Interest: None declared

DOI: 10.1136/annrheumdis-2017-eular.6981

\section{THU0287 CENTRAL ARTERIAL STIFFNESS MEASURED BY THE AUGMENTATION INDEX IS INCREASED IN PATIENTS WITH SYSTEMIC LUPUS ERYTHEMATOSUS (SLE) AND IS DETERMINED BY THE LEVELS OF IGM- $\beta 2-$-GLYCOPROTEIN AND THE SMALL-DENSE HDL PARTICLES}

S. Parra ${ }^{1,2}$, N. Amigo $^{3}$, M. Heras ${ }^{4}$, D. Ibarreche ${ }^{5}$, M. Benavent ${ }^{6}$, A. Navarro ${ }^{6}$, E. Garcés ${ }^{6}$, A. Castro ${ }^{1,2} .{ }^{1}$ Autoimmune diseases unit, Sant Joan's university Hospital; ${ }^{2}$ Institut investigacions Sanitaries Pere i Virgili, Reus, Spain; ${ }^{3}$ Centre for Omic Sciences, Institut Investigacions Sanitaries Pere $i$ Virgili; ${ }^{4}$ Lipid resarch unit (URLA), Rovira i Virgili university; ${ }^{5}$ Unitat de medicina vascular i del metabolisme (UVASMET), Sant Joan University Hospital; ${ }^{6}$ Internal medicine, Sant Joan's university Hospital, Reus, Spain

Background: Patients affected by Systemic Lupus Erythematosus (SLE) show an increase in cardiovascular mortality and morbidity. The accelerated atheroscle- rosis observed in patients with SLE cannot be entirely explained by the traditional cardiovascular risk factors. Patients with SLE show increased subclinical atherosclerosis determined by an increased carotid arterial thickness, endothelial dysfunction and central arterial stiffness. Analysis of lipoproteins by magnetic nuclear resonance (MNR) provide information of those lipoproteins associated with sublcinial atherosclerosis in SLE.

Objectives: To investigate the metabolic and immunological factors associated with the presence of central arterial stiffness determined by the Augmentation Index (Alx) as well as the detailed analysis of the lipid profile performed by magnetic nuclear resonance (MNR)

Methods: Descriptive cross-sectional study of 69 women with SLE compared with a control group of 34 age matched healthy women. On the same day of the study, blood extraction, physical examination and augmentation index (Alx) obtained by Peripheral Arterial Tonometry were performed. The carotid intima-media thickness (IMTc) was also performed on the same day of the study to correlate the arterial stiffness with another subclinical atherosclerosis marker. Analysis of lipoprotein populations by NMR (Liposcale,Biosfer Teslab) were performed.

Results: Patients with SLE showed significant increased arterial stiffness respect the control group $(20.30(21.54) \%$ vs $10.84(11.51) \%, P=0.0021)$ The values of Alx were well correlated with Framingham risk score $(r=0.486, P<0.001)$ as well as the IMTc $(r=0.456, P<0.001)$. The classic cardiovascular risk factors associated with the Alx were the SBP levels $(r=0.456, P<0.001)$ and age $(r=0.456, P$ $<0.001$ ). Patients under antimalarial drugs showed significant decreased Alx (11.74 (11.28) vs 24.97 (20.63); $P=0.024$. Immunological variables associated with Alx were levels of C4 $(r=0.259 ; P=0.046)$ and the IgM- $\beta 2$-glycoprotein levels $(r=0.284, P=0.284)$. As for the lipoprotein populations, Al values correlated with ApoB plasma levels, remnant particles, the number of large, medium and small VLDL particles, the number of small LDL particles and the number of small-dense HDL particles. In the multivariate analysis we found that age $(\beta=0.377(0.117-$ 0.636), $P=0.005)$ IgM-B2-GLP levels $(\beta=0.303(0.026-0.580), P=0.033)$ and the small-dense HDL particles $(\beta=1,463(0.605-2.322), P=0.001)$.

Conclusions: SLE patients show increased central arterial stiffness respect healthy population. Patients treated with antamalarial drugs show lower arterial stifness. In multivariate analyses variables that predicted levels of Alx were age, levels of lgM- $\beta 2$-glycoprotein and the number of small-dense HDL particles.

Disclosure of Interest: None declared

DOI: 10.1136/annrheumdis-2017-eular.3974

\section{THU0288 COMPARISON OF THE CLINICAL, SEROLOGICAL, AND PROGNOSTIC DIFFERENCES AMONG JUVENILE-, ADULT-, AND LATE-ONSET LUPUS NEPHRITIS IN KOREAN PATIENTS: A CASE-CONTROL STUDY}

\section{S.-S. Lee $^{1,1}$, J.-H. Kang, J.-E. Kim, K.-E. Lee, D.-J. Park. Chonnam National} University Medical School and Hospital, Gwangju, Korea, Republic Of

Objectives: SLE patients present with different clinical and serological manifestations according to the age at disease onset. However, it is not known whether there is an association between disease onset age and the clinical presentation of lupus nephritis (LN). Therefore, we investigated whether LN patients could be distinguished based on the time of disease onset and, if so, whether the groups differed in their clinical, laboratory features and long-term prognosis in ethnically homogeneous Korean patients.

Methods: We enrolled 117 SLE patients with available clinical data at the time of renal biopsy for LN from the lupus cohort at Chonnam National University Hospital. We divided the LN patients according to the age at LN diagnosis into three groups [juvenile-onset LN (JLN), diagnosed at $\leq 18$ years; adult-onset LN (ALN), diagnosed at 18-50 years; and late-onset LN (LLN), diagnosed at $>50$ years] and compared the baseline demographic, clinical, histological, and relevant laboratory findings. We also compared the treatment and long-term prognosis of $\mathrm{LN}$ according to those three groups.

Results: Of the 114 LN patients, 20 (17.5\%), 84 (71.8\%), and $13(11.1 \%)$ had JLN, ALN, and LLN, respectively. LLN patients were less educated than ALN and JLN patients $(p<0.001)$. Hypertension and diabetes mellitus at the onset of LN were more common in LLN patients than ALN or JLN patients $(p<0.001$ and $p=0.037$, respectively). Regarding the laboratory findings, LLN patients had a higher white blood cell count and lower eGFR than ALN or JLN patients ( $p<0.011$ and 0.002 , respectively). Histologically, LLN patients had more chronicity indices and a higher chronic score $(p=0.006, p=0.019, p<0.001$ and $p<0.001$, respectively). Anti-Ro antibodies were found more frequently in ALN patients and less frequently in JLN patients $(p=0.024)$ and lower complement levels were more common in JLN patients and less common in LLN patients $(p<0.011$ and 0.002 , respectively). During a mean follow-up of 76.5 months, the development of chronic kidney disease and death from any cause were higher in LLN patients than in JLN and $\operatorname{ALN}$ patients ( $p=0.028$ and $p=0.038$, respectively). Conclusions: LN patients present with different clinical and serological manifestations according to age at disease onset. Interestingly, LLN patients had more chronicity at the time of renal biopsy, and more deterioration of kidney function and death on long-term follow-up, than JLN and ALN patients. Therefore, LLN patients should be monitored and managed carefully to avoid poor outcomes.

Disclosure of Interest: None declared

DOI: 10.1136/annrheumdis-2017-eular.2365 